\title{
Nuclear Archaeology: Verifying Declarations of Fissile-Material Production
}

\section{Steve Fetter ${ }^{a}$}

Controlling the production of fissile material is an essential element of nonproliferation policy. Similarly, accounting for the past production of fissile material should be an important component of nuclear disarmament. This paper describes two promising techniques that make use of physical evidence at reactors and enrichment facilities to verify the past production of plutonium and highly enriched uranium. In the first technique, the concentrations of long-lived radionuclides in permanent components of the reactor core are used to estimate the neutron fluence in various regions of the reactor, and thereby verify declarations of plutonium production in the reactor. In the second technique, the ratio of the concentration of U-235 to that of U-234 in the tails is used to determine whether a given container of tails was used in the production of lowenriched uranium, which is suitable for reactor fuel, or highly enriched uranium, which can be used in nuclear weapons. Both techniques belong to the new field of "nuclear archaeology," in which we attempt to document past nuclear weapons activities and thereby lay a firm foundation for verifiable nuclear disarmament.

\section{INTRODUCTION}

For the first time, the tide of nuclear proliferation-vertical as well as horizontal proliferation-is ebbing. The United States and Russia have agreed to reduce their combined nuclear arsenals from some 50,000 warheads to less than 10,000 by the turn of the century. Just as importantly, Argentina, Brazil, South Africa, Iraq, and North Korea have turned or been turned back from the nuclear threshold.

It is important to ensure that these reductions in weapons and weapon capabilities are as binding as possible. An important means of doing so is

a. School of Public Affairs, University of Maryland, College Park, Maryland 
"nuclear archaeology": to retrospectively document all nuclear weapons activities, especially the production and disposition of fissile materials. Fissile materials-highly enriched uranium (HEU) and plutonium-are the essential ingredients of nuclear weapons, and are by far the most difficult and most expensive part of a nuclear weapon to produce. Indeed, a central part of the nonproliferation regime is based on the control of access to fissile materials through safeguards and export controls. It is vital to bring under international safeguards all fissile materials, starting with those in threshold nations that join the nonproliferation regime, and including all fissile materials from warheads dismantled by the nuclear powers.

The International Atomic Energy Agency (IAEA) requires non-nuclear states to declare the location and quantities of fissile materials when they accept safeguards, but how will we know that they have made complete disclosures? Might not a nation be tempted to reserve enough material to make a dozen bombs "just in case," and might not a fear that material had been withheld inhibit progress toward nuclear disarmament?

The world community now faces this problem in North Korea. North Korea claims to have operated what appears to be a plutonium-production reactor only at low power, and not to have refueled the reactor or to have separated plutonium from spent fuel. Western intelligence analysts are suspicious; some believe that North Korea has already produced a significant quantity of plutonium. Concerns have also been raised about South Africa's declaration of the amount of HEU it produced when it harbored nuclear aspirations. If Israel, India, or Pakistan ever join the Nonproliferation Treaty (NPT) as non-nuclear weapon states, their declarations will no doubt be viewed with similar skepticism. Thus, the problem of verifying declarations of fissile materials is central to the disarmament process.

Verifying such declarations would begin by requesting a detailed history of the production of weapons-grade uranium and plutonium and/or facilities theoretically capable of producing these materials. The original operating records for the production facilities (production reactors and enrichment facilities) could then be examined to ensure that they are consistent with the declaration, and that they are internally consistent. In the case of production reactors, for example, one could request details of the fuel loading (e.g., initial fuel enrichment, masses, and fuel burnup) as a function of time, along with the overall reactor power, energy production, coolant flows and temperatures, and a general description of the reactor. In addition, other records (e.g., mining, milling, purchases, and conversion of natural uranium, fuel fabrication, consumption/production of electricity or heat, and reprocessing activities) could be examined to ensure that they, too, are consistent with the production 
declarations. A parallel set of requirements would apply in the case of enrichment facilities.

All records can be falsified, however. While it is not easy to invent a false operating history that is internally self-consistent, it could be done by a group that was intimately familiar with the production facilities. The authenticity of old records probably could be verified using standard techniques, ${ }^{*}$ but even authentic records can be inaccurate (deliberately or inadvertently). If, however, the records are relatively recent (as would be the case in most of the threshold nations), the operating history is probably stored in computer files, which could be altered easily and without detection.

If, for example, a nation had produced a small but significant quantity of separated plutonium and wished to hide this fact, it could invent a false operating history for the reactor that would show the reactor coming into its present state without being refueled; the spent fuel and reprocessing could be hidden. Similarly, a nation wishing to hide production of HEU could "doctor the books" by claiming instead the production of an amount of low-enriched uranium (LEU) that would have consumed an equivalent amount of uranium feed and separative work.

In most cases, declarations will be made in good faith, and this will be demonstrated by the apparent consistency and authenticity of the records provided. But declarations will be treated with suspicion, and examination of the operating records may fail to resolve (or may even reinforce) these doubts. In these cases it would be helpful to have recourse to physical evidence.

The design characteristics of the facilities, which can be verified by inspection, are unlikely to provide such evidence, since a wide variety of operating histories are reasonably possible at any given facility. Rather, what we seek is evidence about how the facilities were operated in the past. The remainder of this paper investigates two types of evidence-induced radioactivity in production reactors, and the isotopic composition of depleted uranium-that might prove useful in verifying declarations of plutonium and HEU production.

* Old operating records were probably kept on paper, the authenticity of which could be determined by examining the composition of the paper and/or ink as well as the nature of the instrument that applied the ink to the paper. The composition of the fibers in paper changes with time, as does the composition of ink. If the United States had samples of documents from the time period in question on file, they could be compared with the production records. Moreover, production records may contain the signature of an official, which may also be compared with other samples. 


\section{VERIFYING PLUTONIUM PRODUCTION USING INDUCED RADIOACTIVITY IN REACTOR CORES}

Plutonium is produced in nuclear reactors primarily by the absorption of neutrons in U-238:

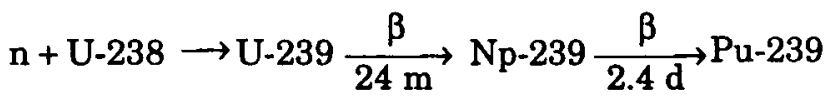

where $\beta$ indicates a type of radioactive decay with the indicated half-life. The probability of this reaction occurring is highest for slow neutrons. Fission of U-235 nuclei in the reactor provides a steady supply or "flux" of neutrons.

Reactors built for the primary purpose of producing plutonium are known as plutonium-production reactors. Production reactors consist of assemblies of fuel and target materials embedded in a moderator. Fission reactions in the fuel produce neutrons which are slowed in the moderator and absorbed in the target. The fuel and target materials may be separate or mixed. The fission of U-235 produces about 2.4 neutrons; in steady-state operation, an average of one of these neutrons causes a new fission, 0.4 are absorbed without causing fission, and about one is absorbed in the target, producing plutonium at the rate of about one gram per megawatt-day.

The principle type of production reactor is the graphite-moderated reactor, fueled with natural or slightly enriched uranium (SEU), which serves as the target as well. The large graphite core is penetrated at regular intervals with a thousand or more horizontal or vertical fuel channels. Heavy-water-moderated reactors have also been used to produce plutonium, using either natural uranium, SEU, or separate HEU fuel and depleted-uranium target assemblies suspended in the water from a steel structure. Since heavy water is a more efficient moderator than graphite, heavy-water reactors are more compact and have higher neutron fluxes than graphite-moderated reactors.

A small fraction of the neutrons produced by fission will be absorbed in the moderator and permanent structural components of the reactor core, usually producing radioactive nuclei. ${ }^{*}$ If these radioactive nuclei have long half-lives (much longer than the lifetime of the reactor), very little radioactive decay will occur, and the concentration of these nuclei will be proportional to the total number of neutrons passing through the material (the neutron "fluence").

* The neutrons also damage reactor materials (e.g., by dislocating atoms and by producing hydrogen atoms). This damage can be correlated with the total neutron fluence, but not as accurately as the concentration of long-lived radionuclides. 
Thus, long-lived radionuclides produced by neutron absorption in naturally occurring nuclei in the permanent components of the reactor core can provide an estimate of the neutron fluence at that point. When coupled with reasonable assumptions about the design and operation of the reactor, measurements of neutron-induced radionuclides at several points in the core can be used to estimate neutron fluences and plutonium production.

The neutron-induced radionuclides chosen for measurement should:

- Have very long half-lives (at least 100 years) so that the total production is independent of time.

- Not exist in significant concentrations in nature so that one can be sure that they are produced in the reactor (this eliminates, for example, $\mathrm{K}-40$ and $\mathrm{Rb}-87$ ).

- Not be noble gases, which often escape from reactor materials.

- Not be fission products, actinides, or their decay products, because reactor components might be contaminated with these.

- Be produced in slow-neutron reactions with naturally occurring isotopes.

These conditions are very restrictive. Only 67 radionuclides that do not exist in nature have half-lives greater than 100 years; of these, two are noble gases, 23 are in the range of atomic weights produced during fission (79 to 166), and 23 are actinides or decay products of actinides. Nine of the remaining 19 radionuclides are not produced, either directly or as decay products, from slowneutron reactions with naturally occurring isotopes. The ten remaining radionuclides that meet all five criteria, along with the elements from which these radionuclides are produced in thermal-neutron reactions with naturallyoccurring isotopes, are listed in table 1.

To be useful, a radionuclide must meet an additional criterion: the element from which it is produced must be present in significant concentrations in a permanent component of the reactor core. The most common production reactor core materials are graphite (in graphite-moderated reactors) and some type of metal-steel, aluminum, zircalloy, etc. (in all reactors). The concentrations of elements listed in table 1 in samples of the graphite used in the construction of the original Hanford reactors are given in table 2. Also given in table 2 is a range of concentrations of these elements in certain reactorgrade steels.

At first glance, $\mathrm{C}-14$ might appear to be the best radionuclide to measure in graphite. The presence of nitrogen in concentrations as low as one part per million (ppm) would, however, make an accurate estimate of the neutron flu- 
Table 1: Radionuclides that: (1) have half-lives greater than 100 years (2) do not exist in significant quantities in nature ${ }^{a}$ (3) are not noble gases (4) are not fission products or actinides, and (5) are produced from slow-neutron reactions with naturally occurring isotopes.

$\begin{array}{ll}\text { Radlonuclide } & \text { Produced from isotope of } \\ \mathrm{Be}-10 & \text { Beryllium } \\ \mathrm{C}-14 & \text { Carbon, Nitrogen, Oxygen } \\ \mathrm{Cl}-36 & \text { Chlorine, Potassium } \\ \mathrm{Ca}-41 & \text { Calcium } \\ \mathrm{Ni}-59 & \text { Nickel } \\ \mathrm{Ni}-63 & \text { Nickel. Znc } \\ \mathrm{Re}-186 \mathrm{~m} & \text { Rhenium } \\ \mathrm{Ir}-192 \mathrm{~m} 2 & \text { Iridium } \\ \mathrm{Pb}-205 & \text { Lead } \\ \mathrm{Bi}-210 \mathrm{~m} & \text { Bismuth } \\ \text { and } \mathrm{C}+36 \text { are present in minute concentrations in noture. }\end{array}$

a. Be-10, C-14, and $\mathrm{Cl} 36$ are present in minute concentrations in nature.

ence impossible, since the concentration of nitrogen would vary during the life of the reactor, and since eight ppm of nitrogen generates as much C-14 as does the one percent C-13 in the carbon. Calcium-41 and Ni-59 seem better suited for measurement because calcium and nickel are present in very pure graphite, and because both have long half-lives, well-known reaction cross sections, and are produced in quantity by only one reaction. In the case of steel, Ni-59 and $\mathrm{Ni}-63$, which are produced by reactions with nickel, are the best candidates for measurement.

Table A-2 in the appendix gives estimates of the concentrations of these radionuclides that could be expected in the graphite or steel components of production-reactor cores. One should be able to measure the radionuclide concentrations in table A-2, which range from $10^{-12}$ to $10^{-4}$, without too much trouble. ${ }^{1}$ Portable mass spectrometers accurately measure concentrations smaller than $10^{-9}$ (one ppb); larger machines can go much lower. Concentra- 
Table 2: Concentrations of key elements in samples of graphite and steel used in the construction of the original Hanford reactors.

Element

Beryllium

Carbon

Nitrogen

Oxygen

Chlorine

Połassium

Calcium

Nickel

Zinc

Rhenium

Iridium

Lead

Bismuth

\section{Concentration}

(in ppm unless otherwise indicated)

Graphitea $^{a}$

Steel ${ }^{b}$

$<0.0005$

$100 \%$

$2,000-5,800$

$10-100$

$40-190$

$$
<50
$$

$<0.02$

$0.13-210$

$0.02-2.5$

$0.5-16 \%$

$0.06-160$

$$
<0.01
$$

a. Wiliam Morgan, personal communication.

b. Data for six samples of steel of the types SS-316. HT-9, and Fe-1422 from Steve Fetter. "The Radiological Hazards of Magnetic Fusion Reactors." Fusion Technotogy 11 (2) March 1987.

tions of $\mathrm{C}-14$ as small as $10^{-15}$ are routinely measured with high accuracy. Although radionuclide concentrations and neutron cross sections can be measured with an accuracy of a few percent, uncertainties in the operating record, the reactor design, and the computer model of the reactor probably limit the overall accuracy of the estimated neutron fluence, and the corresponding esti-

* The concentration of C-14 in contemporary carbon is 1.3 parts per trillion (ppt); C14 dating has been used to establish ages of over 50,000 years, which corresponds to a C-14 concentration of less than $1.3 \exp (-50,000 / 8,270)=0.003$ ppt. 
Table 3: Isotopes of natural uranium. ${ }^{a}$

\begin{tabular}{|c|c|c|c|c|}
\hline \multirow[t]{2}{*}{ Isotope } & Mass & \multicolumn{2}{|c|}{$\begin{array}{l}\text { Concentration } \\
\text { in natural uranium }\end{array}$} & Half-llfe \\
\hline & $a m u$ & atom $\%$ & weight $\%$ & years \\
\hline $\mathrm{U}-234$ & 234.040904 & 0.0055 & 0.0054 & $2.454 \times 10^{5}$ \\
\hline U-235 & 235.043915 & 0.7200 & 0.7110 & $7.037 \times 10^{8}$ \\
\hline U-238 & 238.05077 & 99.2745 & 99.2836 & $4.468 \times 10^{9}$ \\
\hline
\end{tabular}

mate of plutonium production, to about 10 percent.*

Although the total neutron fluence can be verified in this way, a key problem is that it is virtually impossible to distinguish between the production of plutonium and other isotopes such as tritium. ${ }^{\dagger}$ The excess neutrons produced by fission can be used to produce either Pu-239 (from U-238 targets) or tritium (from lithium-6 targets). This complication arises primarily in deuteriummoderated reactors, which (at least in the United States) have been used to produce both tritium and plutonium. One can only determine whether the declared production of both materials in a given reactor is consistent with the estimated neutron fluence.

\section{VERIFYING HEU PRODUCTION USING THE ISOTOPIC CONCENTRATION OF THE TAILS}

Natural uranium contains three isotopes: U-238, U-235, and U-234 (see table 3 ). Uranium- 235 is the only naturally occurring nuclide that can sustain a fast-fission chain reaction; it is present in natural uranium at a concentration

* An accuracy of 10 percent may seem optimistic, but one should bear in mind that details of the reactor design and details of the operating record would be provided. If these records are complete and genuine, there is no reason that the inspector's estimate of the plutonium production would not be as accurate as the inspectee's, which typically are accurate to within several percent.

$\dagger$ US production reactors have also been used to produce other isotopes, such as Po210, U-233, Pu-238, Cm-244, and Cf-252, but in quantities negligible compared to that of $\mathrm{Pu}-239$. 
of 0.711 percent by weight. Enrichment processes increase the concentration of U-235. Uranium has been produced with several different U-235 concentrations for a wide variety of uses. In the United States, for example, slightly enriched uranium $(\approx 1$ percent $U-235)$ was used to fuel the graphite plutonium-production reactors at Hanford; low-enriched uranium (2-4 percent U235 ) is used to fuel commercial light-water reactors; and highly enriched uranium $(90+$ percent $U-235)$ is used to fuel research and test reactors, the heavywater production reactors at Savannah River, naval reactors, and in nuclear weapons.

Weapons-grade HEU typically is 93 percent U-235, although somewhat lower concentrations are also usable in the fissile components of nuclear weapons. The concentration of U-235 is increased from 0.711 to 93 percent in an enrichment facility, which separates molecules containing the lighter U-235 atom from those containing the U-238 atom. The stream of natural uranium entering the enrichment facility is called the "feed," the exiting stream enriched in U-235 is called the "product," and the exiting stream depleted in U-235 is called the "tails." The tails remain behind, but 1,000 kilograms of tails (containing 0.2 percent $U-235$ ) could have been the result of the production of 5.5 kilograms of HEU (93 percent U-235), 220 kilograms of LEU (3 percent U-235), or 640 kilograms of SEU (1 percent U-235).

Various techniques have been employed to separate U-235 from U-238, the most common being gaseous diffusion and gas centrifugation; aerodynamic and electromagnetic separation have seen more limited use. Because the mass of U-235 is so close to that of U-238, the amount by which the concentration of U-235 can be increased in any process typically is quite small, and hundreds of stages must be connected in series to obtain even low-enriched uranium. ${ }^{*}$ By counting the number of such stages, one should be able to determine whether the facility is capable of producing HEU. Unfortunately, a facility capable of producing a small flow of HEU could be converted to one capable of producing a large flow of LEU (or vice versa), and this conversion might not be detected.

One key to distinguishing the production of HEU from the production of LEU lies in the concentration of the minor isotope U-234 in the tails. If uranium contained only U-235 and U-238, the concentration of each isotope in the feed to stage $s$ could be matched exactly to the concentration in the tails of stage $s+1$. This minimizes separative work, since uranium with unequal iso-

* The exception is electromagnetic separation, in which the separation factor is so high that only one or two stages are required to produce weapons-grade HEU. In addition, the most modern centrifuges have relatively high separation factors that require tens, rather than hundreds, of stages. 
topic compositions is never mixed, and the tails contain no information about the product. Such matching is impossible with three isotopes. If we match the concentrations of U-235 and U-238, then the concentration of U-234 in the tails from the second enriching stage will be lower than the concentration of U-234 in the feed to the first enriching stage (and so on up the enriching section of the cascade). Since the tails of the first enriching stage is the feed for the first stripping stage, the greater rate of U-234 separation-which depends on the length of the enriching section-will show up in lower U-234 concentrations in the tails of the cascade.

If the difference in the U-234 concentration is large enough, it should be possible to determine whether a given container of tails was used in the production of HEU or LEU." Inspections of tails might be able to determine whether a nation claiming to have produced only LEU had in fact produced some amount of HEU. Moreover, if all the tails from the enrichment process are available for inspection, this technique could be used to determine the total production of LEU and HEU.

Appendix B shows how one can calculate the concentration of U-234 in the tails as a function of the concentration of U-235 in the product and the tails. Figure 1 shows a plot of the U-235: U-234 concentration ratio in the tails as a function of U-235 enrichment, for tails concentrations of 0.2 and 0.3 percent U-235, assuming natural uranium feed. Note that this ratio is 20 to 30 percent greater for HEU compared to SEU, and 10 to 12 percent compared to LEU-a difference that should be easily detectable. ${ }^{2}$

The story is not so simple, however. One problem is that the concentration of U-234 in natural uranium ore is not a universal constant, as implied in table 3. Although the relative concentration by weight of U-235 in natural uranium varies by less than a tenth of a percent from sample to sample, ${ }^{3}$ the concentration of U-234 can vary by more than 10 percent from sample to sample. ${ }^{4}$ To understand why this is so, consider the decay chain that leads to production of U-234:

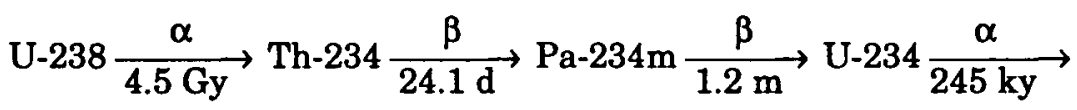

Since the Earth is billions of years old, we would expect that the relative concentrations of U-234 and U-238 would be given by the ratio of the half-

There may be ways to disguise this information, but these would be highly burdensome and could themselves risk detection. 


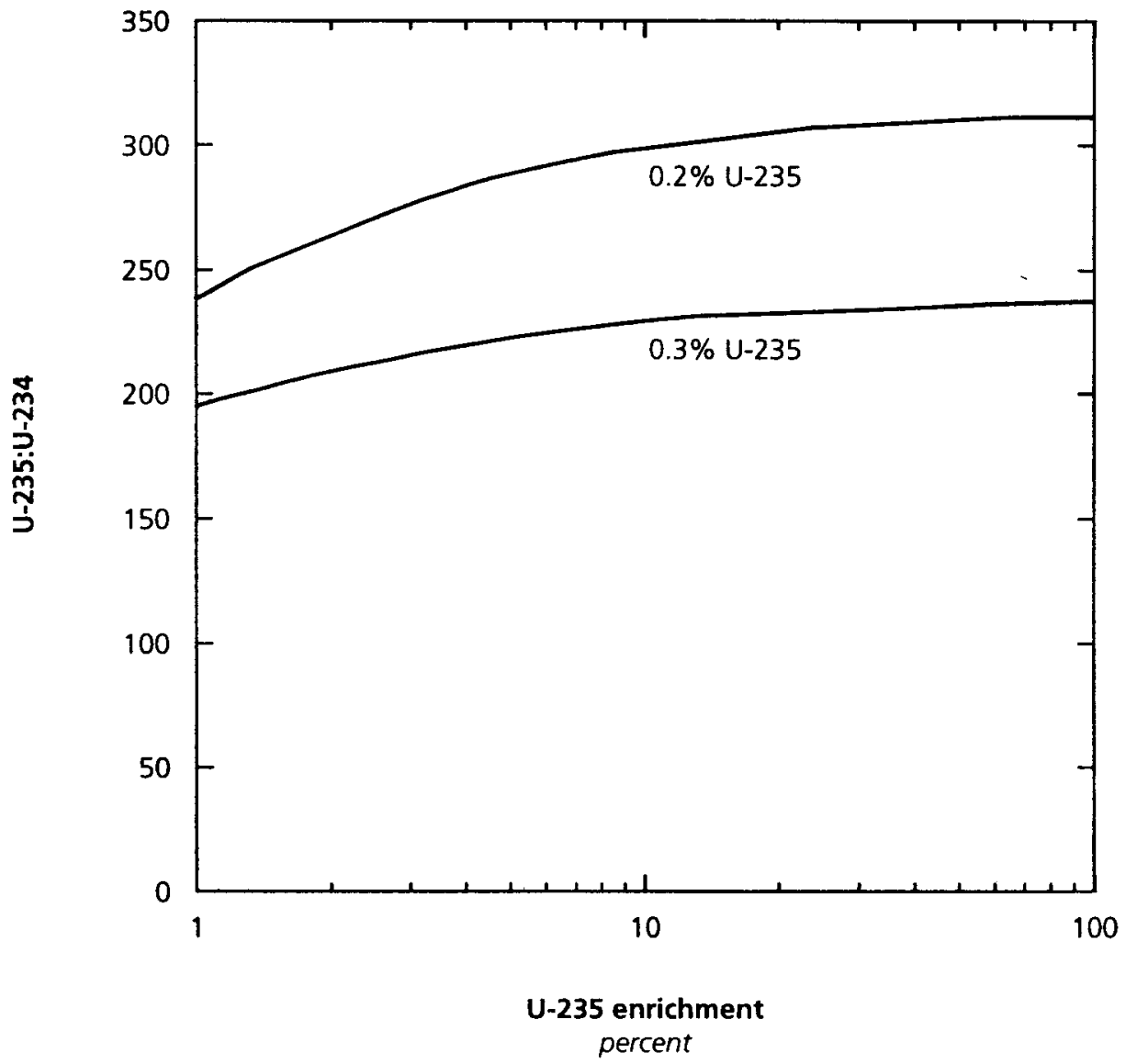

Figure 1: The U-235:U-234 ratio in the tails as a function of the U-235 enrichment of the product, for $U-235$ tails concentrations of 0.2 percent and 0.3 percent.

lives, which should give a U-234 concentration of $54.5 \mathrm{ppm}$ in natural uranium. But the half-lives of Th-234 and Pa-234m allow time for the chemical removal of the more mobile thorium and protactinium from the uranium. For this reason, the U-234: U-238 activity ratio is $1.14 \pm 0.01$ in the oceans, while on land it is less than one. Data of Smith and Jackson indicate activity ratios of 0.914 to 0.985 in natural uranium from 16 widely distributed sources. ${ }^{5}$

Uncertainties in the U-234 concentration of natural uranium do not necessarily negate the usefulness of this technique, however. If the feed used to produce HEU was taken from the same source as that used to produce LEU, as has probably been the case in smaller enrichment enterprises, then the U235 : U-234 ratio can be used to distinguish between the two types of tails. 
And even if the HEU and LEU feeds came from quite different sources, one could salvage the technique by measuring the U-234 concentration in a sample of the HEU or LEU products, or by directly measuring the U-234 concentrations in the uranium ores used for feed.

Another complication is the possibility that more than one product stream was withdrawn from the enrichment cascade, as has been the case for the large cascades in the United States. If, for example, LEU is withdrawn from the cascade while HEU is produced, the U-234 concentration in the tails would assume some intermediate value. Without additional information about the operation of the cascade (e.g., the relative amounts of the two streams produced) it would be impossible to deduce the product enrichments from the tails. One could, however, verify that the tails assay was consistent with the production records, even though other production histories might also be consistent with the tails assay.

A final complication is the possibility that uranium from reprocessed reactor fuel was used as feed. ${ }^{6}$ In this case, several other long-lived isotopes of uranium will also be present in the feed: U-232, U-233, and U-236. The most important of these is U-236, which is formed by neutron capture with U-235. If, however, the composition of the feed is known (from records or estimates), there should no problem in using the technique outlined in appendix B to estimate enrichments.

Perhaps the most severe limitation in using tails to determine past enrichment practices is that not all of the tails may be available for inspection. If, for example, one knew which tails were used to produce HEU, these could be hidden and the production records (and electricity production/consumption records) falsified. In the United States, depleted uranium has been used for ballast, bullets, nuclear weapon components, and reactor targets, although these have accounted for a relatively small fraction of all tails. Hiding a large fraction of the tails would not be a small task; concealing 10 percent of US HEU production would, for example, require hiding 100,000 tons of depleted uranium. Moreover, large stockpiles of depleted uranium might be detected by aerial radiation surveys.

The verification of production records might be facilitated by dating the tails to determine the year they passed through the cascade. In this way, a cheater would have to eliminate the tails produced during a certain time period in order for the physical evidence to agree with falsified production records. ${ }^{*}$ The best clock is provided by the decay of U-235, which results in the

* Once again, there might be a fairly straightforward but burdensome way to disguise this information. 
buildup of Pa-231 and Ac-227:

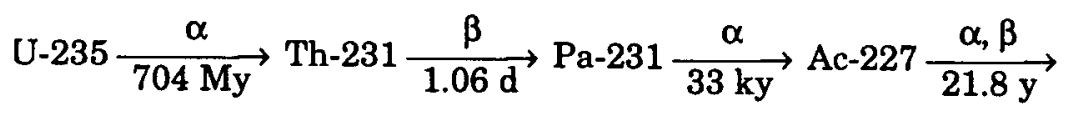

A similar internal clock is provided by the decay of the less abundant U-234 to Th-230.

\section{CONCLUSIONS}

Accounting for the past production of fissile materials is a vital element in the worldwide movement toward nuclear disarmament. Countries turning away from the nuclear threshold may want to demonstrate that they have not produced fissile materials or that all such materials have been put under international safeguards.

Declarations of fissile material production, supplemented by facility specifications and original production records, are an essential part of this task, but declarations and production records can be falsified. If suspicions arise, it would be helpful to have access to physical evidence that could verify the accuracy of the declarations. North Korea's claim that it has produced no plutonium provides a vivid example of the sorts of suspicions that can surround such declarations, even when supplemented with inspections and records.

In the case of plutonium production, the buildup of long-lived radionuclides in the permanent components of the reactor core provides such evidence. Measurements of the concentrations of these radionuclides at several dozen points in the reactor core, coupled with a model of the core, could provide an independent verification of the operating records. This technique is sufficiently promising that serious consideration should be given to doing a full-scale demonstration using a US or Russian production reactor or similar civilian reactor.

Verifying HEU production is more difficult because enrichment facilities have been used to produce uranium of varying enrichments. LEU is unusable for bombs, but almost any facility capable of producing LEU is theoretically capable of producing HEU. If a nation claims to have produced only LEU, one could verify this by measuring the U-235 : U-234 ratio in the tails, assuming that the tails are available for inspection and that the composition of the feed is known. The date that the tails were produced can be estimated by measuring the concentration of decay products of uranium isotopes, thus providing 
additional data that can be used to verify the accuracy of production records.

Nuclear archaeology is a field with tremendous potential for growth, encompassing but going well beyond traditional safeguards techniques. This paper only scratches the surface of this new field, but I hope it will stimulate additional research that will help nations to gain confidence in the disarmament process.

\section{ACKNOWLEDGEMENTS}

The author would like to thank Frank von Hippel, Jim Powell, Allen Groff, Willy Higinbotham, and Marvin Miller for their helpful comments. Funding for this research was provided by the Federation of American Scientists and the University of Maryland Office of Graduate Studies and Research.

\section{Appendix A: The Production of Radionuclides in Reactor Cores}

The buildup of radionuclide $y$ from neutron reactions with a stable isotope $x$ can be described by the following equations

$$
\begin{gathered}
\frac{d N_{y}}{d t}=N_{x} \sigma_{x y} \phi-N_{y} \sigma_{y} \phi-N_{y} \lambda \\
\frac{d N_{x}}{d t}=-N_{x} \sigma_{x} \phi
\end{gathered}
$$

where $N_{x}$ and $N_{y}$ are the atomic concentrations of the stable isotope and the radionuclide, $\sigma_{x}$ and $\sigma_{y}$ are the neutron cross sections for transmuting $x$ or $y$ into some other nucleus and $\sigma_{x y}$ is the cross section for transmuting $x$ into $y\left(\mathrm{~cm}^{2}\right), \phi$ is the neutron flux (neutrons $\mathrm{cm}^{-2} \mathrm{sec}^{-1}$ ), and $\lambda$ is the decay constant of the radionuclide $\left(\mathrm{sec}^{-1}\right.$ ). For the cases considered here, we can ignore other reactions that may produce or deplete $x$ and $y$, and we can therefore set $\sigma_{x y}=\sigma_{x}$. Solving equation A-1b for $N_{x}$ and substituting its value into equation $\mathrm{A}-1 \mathrm{a}$, we have

$$
\frac{d N_{y}}{d t}=N_{x}^{0} \sigma_{x} \phi \exp \left(-\sigma_{x} \Phi\right)-N_{y} \sigma_{t} \phi-N_{y \lambda}
$$

where $N_{x}^{o}$ is the initial concentration of isotope $x$, and $\Phi$, the integrated neutron fluence (neutrons $\mathrm{cm}^{-2}$ ), is given by

$$
\Phi=\int_{0}^{t} \phi(\tau) d \tau
$$


If $\lambda \ll<\sigma_{y}$, or if $\phi$ is roughly constant, equation $A-2$ can be solved to give

$$
N_{y} \approx \frac{N_{x}^{0} \sigma_{x}}{\sigma_{y}-\sigma_{x}+(\lambda T / \Phi)}\left[\exp \left(-\sigma_{x} \Phi\right)-\exp \left(-\sigma_{y} \Phi-\lambda T\right)\right]
$$

where $T$ is the total time that the material was exposed to the neutron flux. For the neutron fluences and reactor operation times of interest here, this equation will give reasonably accurate results if the radionuclide has a half-life of at least 100 years. Note that if $\sigma_{x} \Phi \ll 1$ and $\sigma_{y} \Phi \ll 1$, then $N_{y} \approx N_{x}^{0} \sigma_{x} \Phi$.

The neutron flux in graphite reactors is typically about $10^{13}$ neutrons $\mathrm{cm}^{-2} \mathrm{sec}^{-1}$; the flux in heavy-water reactors is roughly an order of magnitude greater, and the flux in both types of reactors is predominately thermal. If a production reactor has operated for 40 years at a capacity factor of 0.5 , the integrated thermal-neutron fluence would be roughly $10^{22}$ to $10^{23}$ neutrons $\mathrm{cm}^{-2}$; reactors operated for a single production cycle would experience fluences two orders of magnitude smaller. Using the data in tables 2 and A-1, the range of concentrations of long-lived radionuclides in graphite and steel can be estimated; the results are given in table A-2.

The actual radionuclide concentrations are affected slightly also by the resonance capture of epithermal neutrons. If the concentrations of two or more radionuclides are measured, and if the target nuclei from which they are produced have widely varying ratios of the resonance-capture integral to the thermal cross section, then the ratio of the epithermal to thermal neutron flux could be estimated. ${ }^{7}$ This would allow moreaccurate estimates of plutonium production, since about 25 percent of plutonium production in graphite and heavy-water reactors is due to the capture of epithermal neutrons."

* In a heavy-water reactor fueled with natural uranium, about 75 percent of the plutonium produced after low burnups $\left(\approx 700 \mathrm{MWd} \mathrm{te}^{-1}[\mathrm{U}]\right)$ is due to the absorption of thermal neutrons in U-238; the remainder is produced by the absorption of epithermal and, to a lesser extent, fast neutrons. See M. Benedict, T.H. Pigford, and H.W. Levi, Nuclear Chemical Engineering (New York: McGraw-Hill, 1981) pp. 137-156. The fraction of plutonium produced with epithermal neutrons in a graphite production reactor should be similar. 
Table A-1: Half-lives and cross sections of the radionuclides listed in table 1, and the reactions and cross sections, and abundances of the isotopes from which they are produced. ${ }^{a}$

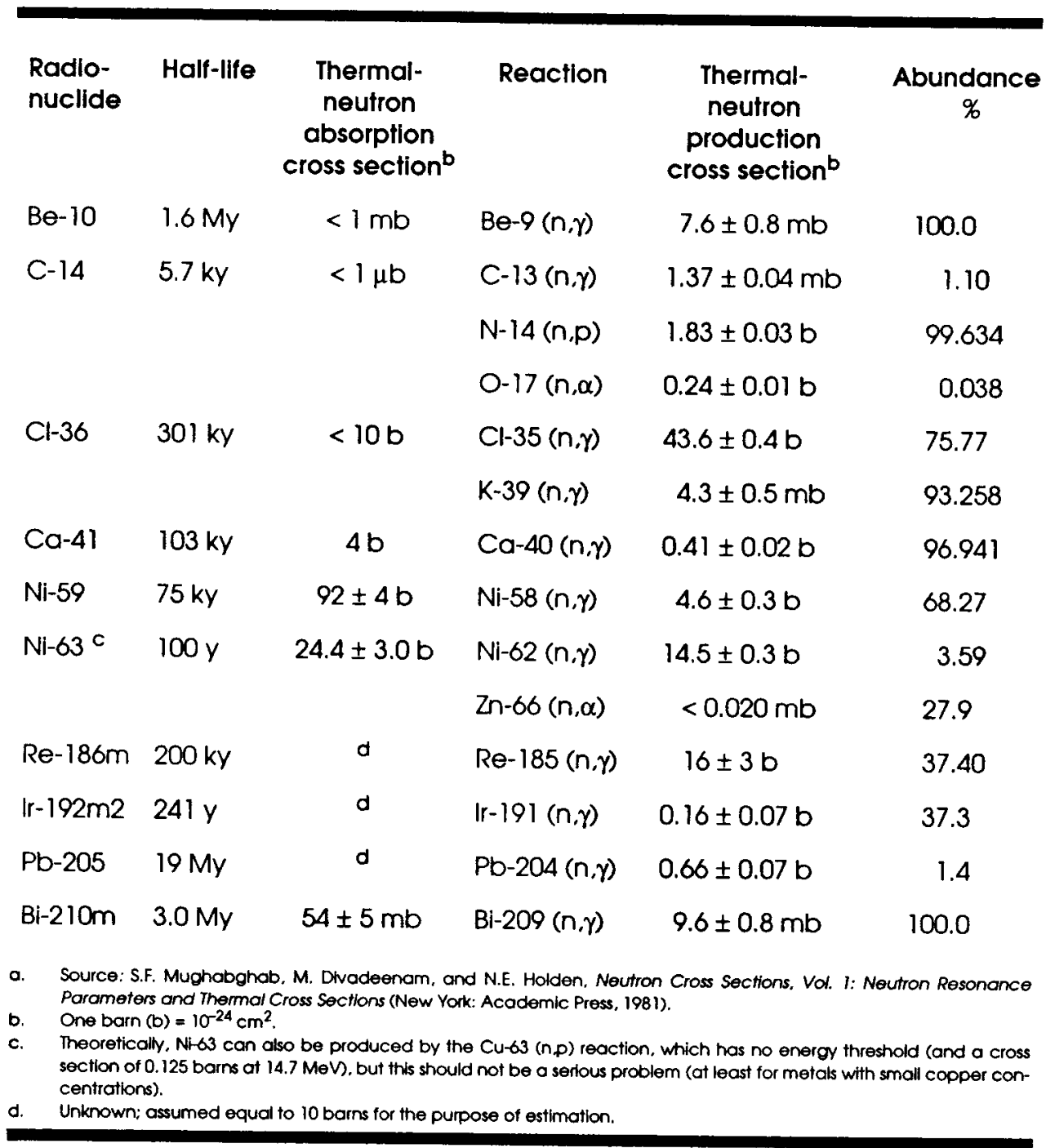


Table A-2: Concentrations of long-lived radionuclides expected in graphite and steel components of reactor cores, assuming thermal-neutron fluences of $10^{20}$ to $10^{23} \mathrm{n} \mathrm{cm}^{-2}$.

\section{Radionuclide concentration}

in graphite

$\begin{array}{llcccr}\text { Radionuclide } & \begin{array}{l}\text { Produced } \\ \text { trom }\end{array} & \text { Low } & \text { High } & \text { Low }^{\mathbf{b}} & \text { High }^{\mathbf{b}} \\ \mathrm{Be}-10 & \mathrm{Be}-9 & & 0.04 \mathrm{ppt} & & \\ \mathrm{C}-14 & \mathrm{C}-13 & 2 \mathrm{ppb} & 2 \mathrm{ppm} & 3 \mathrm{ppt} & 9 \mathrm{ppb} \\ & \mathrm{N}-14 & 20 \mathrm{ppb} & 20 \mathrm{ppm} & 7 \mathrm{ppb} & 30 \mathrm{ppm} \\ & \mathrm{O}-17^{\mathrm{C}} & & 10 \mathrm{ppt} & & 10 \mathrm{ppt}\end{array}$

$\mathrm{Cl}-36$

$\mathrm{Cl}-35$

K-39

Ca-41

Ca-40

Ni-59

Ni-58

$\mathrm{Ni}-62$

Zn-66

Re-186m

Re-185 C

$\mathrm{Ir}-192 \mathrm{~m} 2$

$|r-19|^{C}$

Pb-205

$\mathrm{Pb}-204$

$\mathrm{Bi}-209$

Bi-210m
20 ppm

5 ppt

$0.04 \mathrm{ppt}$

$0.7 \mathrm{ppb}$

5 ppt $\quad 7$ ppm

$4 \mathrm{ppt} \quad 60 \mathrm{ppb}$

1 ppm

400 ppm

0.8 ppt

$20 \mathrm{ppb}$

0.2 ppm

100 ppm

30 ppt

1 ppb

$1 \mathrm{ppb}$

4 ppt

$4 \mathrm{ppt}$

$6 \mathrm{ppt}$

a. Using the lowest concentrations in table 2, o thermatneutron fluence of $10^{20} \mathrm{~cm}^{-2}$, and ignoring resonance capture.

b. Using the highest concentrations in table 2, a thermatneutron fluence of $10^{23} \mathrm{~cm}^{-2}$, and ignoring resonance capture.

c. Concentration in graphite or steel not listed in table 2; set equal to 1 ppm for oxygen and the overage crustal abundance for $R e(5 \mathrm{ppb})$ and ir (1 ppb) to give order-of-magnitude estimates. 


\section{Appendix B: The Isotopic Composition of Depleted Uranium}

Let $x, y$, and $(1-x-y)$ be the concentrations of $U-235, \mathrm{U}-234$, and $\mathrm{U}-238$, let $M$ be the mass of uranium, and let the subscripts $f, p$, and $t$ denote the feed, product, and tails. The flows into and out of the enrichment facility are as follows:

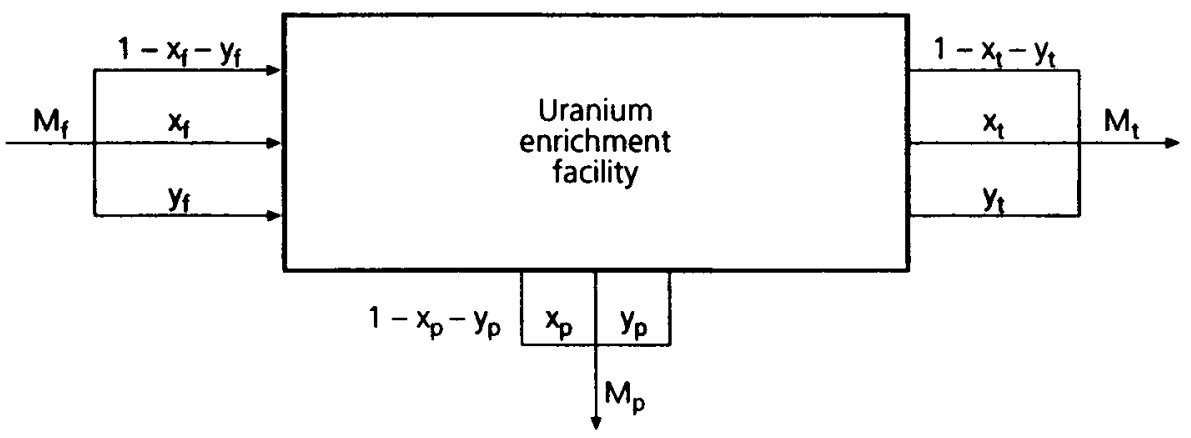

A mass balance gives three equations

$$
\begin{gathered}
M_{f}=M_{p}+M_{t} \\
x_{f} M_{f}=x_{p} M_{p}+x_{t} M_{t} \\
y_{f} M_{f}=y_{p} M_{p}+y_{t} M_{t}
\end{gathered}
$$

The additional required equation is derived from the value function for a threecomponent mixture. For an ideal symmetric cascade in which the concentration of $U$ 235 in the feed to stage $s$ is matched to the concentration of U-235 in the tails from stage $s+1$, the value function, in its most general form, is given by ${ }^{8}$

$$
V(x, y)=c_{0}+c_{1} x+c_{2} y+c_{3} H(x, y)+U(x, y)
$$

where $c_{0} c_{1}, c_{2}$, and $c_{3}$ are constants, and where the functions $H(x, y)$ and $U(x, y)$ are given by 


$$
\begin{gathered}
H(x, y)=y R^{-(2 k-1)} \\
U(x, y)=\left[2 x+\frac{2 k(y-1)}{2 k-1}\right] \ln R \quad(2 \mathrm{k} \neq 1)
\end{gathered}
$$

$R$, the isotope abundance ratio for $x$, is given by

$$
R=\frac{x}{1-x-y}
$$

and $k$, the ratio of the separation factors, is given by

$$
k=\frac{g_{y}}{g_{x}}=\frac{\alpha_{y}-1}{\alpha_{x}-1}
$$

where $g_{y}$ and $g_{x}$ are the separation gains for U-234 and U-235 and $\alpha_{y}$ and $\alpha_{x}$ are the separation factors for U-234 and U-235. For gaseous diffusion with $\mathrm{UF}_{6}{ }^{9}$

$$
\alpha_{y} \approx\left[\frac{352}{348}\right]^{1 / 2} \quad \alpha_{z} \approx\left[\frac{352}{349}\right]^{1 / 2} \quad k=1.33645 \approx \frac{4}{3}
$$

The separative work of a stream is the product of the mass times its value; since the separative work of the cascade equals the sum of the separative work of the stages, it follows from equation B-4 that ${ }^{10}$

$$
\sum_{j=1}^{J} M_{j} H\left(x_{j}, y_{j}\right)=0
$$

where the $M_{j}$ are the masses (or flow rates) of the $J$ stream flows from the cascade. Substituting equation B-5 into equation B-10 and setting $k=4 / 3$, we have

$$
\frac{M_{p} y_{p}}{\left(\frac{x_{p}}{1-x_{p}-y_{p}}\right)^{5 / 3}}+\frac{M_{t} y_{t}}{\left(\frac{x_{t}}{1-x_{t}-y_{t}}\right)^{5 / 3}}+\frac{M_{p} y_{f}}{\left(\frac{x_{f}}{1-x_{f}-y_{f}}\right)^{5 / 3}}=0
$$

for the cascade's three flows. To eliminate the masses, we divide equation B-11 by $M_{f}$, and solve equations B-1 and B-2 for $\left(M_{p} / M_{f}\right)$ and $\left(M_{t} / M_{f}\right)$ 


$$
\begin{aligned}
& \frac{M_{p}}{M_{f}}=\frac{x_{f}-x_{t}}{x_{p}-x_{t}} \\
& \frac{M_{t}}{M_{f}}=\frac{x_{p}-x_{f}}{x_{p}-x_{t}}
\end{aligned}
$$

The remaining unknown, $y_{p}$, can be cast as a function of $x_{p}$ using equations B-1, B-2, and B-3

$$
y_{p}=\left(\frac{x_{p}-x_{t}}{x_{f}-x_{t}}\right)\left(y_{f}-y_{t}\right)+y_{t}
$$

Substituting equations B-12, B-13, and B-14 into equation B-11, we have the following equation for $x_{p}$ in terms of the four known variables $x_{f}, x_{t}, y_{f}$, and $y_{t}$

$$
\frac{\left(\frac{x_{f}-x_{p}}{x_{p}-x_{t}}\right) y_{t}+y_{f}}{\left[\frac{x_{p}}{1-x_{p}-\left(\frac{x_{p}-x_{t}}{x_{f}-x_{t}}\right)\left(y_{f}-y_{t}\right)-y_{t}}\right]^{5 / 3}}+\frac{\left[\frac{x_{p}-x_{f}}{x_{p}-x_{t}}\right] y_{t}}{\left[\frac{x_{t}}{1-x_{t}-y_{t}}\right]^{5 / 3}}=\frac{y_{f}}{\frac{x_{f}}{1-x_{f}-y_{f}}}
$$

Unfortunately, this equation cannot be solved analytically for $x_{p}$ (even if we assume that $\mathrm{y} \ll 1$ ). We can, however, solve the equation numerically using the values of $x_{f}$ and $y_{f}$ for natural uranium given in table 3 . Table B-1 gives values of concentration of U-234 in the tails $\left(y_{t}\right)$ as a function of the enrichment of the product $\left(x_{p}\right)$ for two common tails assays ( $x_{t}=0.2$ and 0.3 percent).

If another flow is added to the cascade (e.g., two product flows), this can be accommodated by adding the terms $M_{q}, x_{q} M_{q}$, and $y_{q} M_{q}$ to the right-hand side of equations B-1, B-2, and B-3, and the term $y_{q} M_{q}\left[x_{q} /\left(1-x_{q}-y_{q}\right)\right]^{-5 / 3}$ to the left-hand side of equation B-11.

Mixtures with more than three isotopes (e.g., from reprocessed uranium feed) are straightforward to solve, since each additional component gives rise to an equation similar to equation B-10 but with a new value of $k$ (corresponding to the ratio of the separation gains of the new component to that of U-235). ${ }^{11}$ If the composition of the feed is known (from records or estimates), there should no problem in using this procedure to estimate enrichments. 
Table B-1: The concentration of U-234 in the tails $\left(y_{t}, p p m w\right)$, as a function of the concentration of U-235 in the product ( $x_{p}$. percent by weight) for concentrations of $U-235$ in the tails $\left(x_{t}\right)$ of 0.2 and 0.3 percent by weight, for $k=1.33645$.

\begin{tabular}{cccc}
\hline$x_{p}$ & \multicolumn{2}{c}{$y_{f}$ (ppmw) } & Fuel \\
$\%$ & $x_{f}=0.2 \%$ & $x_{f}=0.3 \%$ & \\
1 & 8.27 & 15.3 & SEU \\
2 & 7.51 & 14.3 & \\
3 & 7.19 & 13.8 & LEU \\
4 & 7.02 & 13.6 & \\
10 & 6.66 & 13.0 & \\
20 & 6.52 & 12.8 & \\
40 & 6.45 & 12.70 & \\
60 & 6.42 & 12.66 & \\
80 & 6.410 & 12.64 & \\
90 & 6.405 & 12.63 & weapons-grade \\
98 & 6.402 & 12.62 & naval-grade \\
\hline
\end{tabular}

The time elapsed since the tails were produced can be determined by measuring the buildup of radioactive decay products in the uranium. If the parent (U-235) is present at concentration $C_{1}(0)$ after enrichment, and if none of the other members of the decay chain are initially present (a good assumption), then the concentration of the $i$ th nuclide at time $t$ after enrichment is given by

$$
C_{i}(t)=C_{1}(0) \prod_{j=1}^{i-1} \lambda_{j} \sum_{h=1}^{i} \frac{\exp \left(-\lambda_{h} t\right)}{\prod_{\substack{p=1 \\ p \neq h}}^{i}\left(\lambda_{p}-\lambda_{h}\right)}
$$

where $\lambda_{j}$ refers to the decay constant of the $j$ th nuclide. 


\section{NOTES AND REFERENCES}

1. I have also explored the possibility of measuring the concentration of radionı clides in graphite in situ by moving a germanium detector through the fuel channe. and measuring the intensity of the characteristic gamma rays emitted during tr. decay of these radionuclides. Unfortunately, only one of the ten radionuclides, $\mathrm{Bi}-21$ । emits energetic gamma rays (strong gamma rays at 266,305 , and $650 \mathrm{keV}$ ); $\mathrm{Be}-10, \mathrm{C}$ 14 , and $\mathrm{Ni}-63$ emit no photons, $\mathrm{Cl}-36, \mathrm{Ca}-41, \mathrm{Ni}-59$, and $\mathrm{Pb}-205$ emit only $\mathrm{x}$-rays, an Re-186m and Ir-192m2 emit weak, low-energy gamma rays. Bismuth-210m is pri duced by $(n, \gamma)$ reactions with the sole naturally occurring isotope of bismuth, Bi-20! Since the concentration of bismuth in samples of Hanford graphite material was belor the measurable (but unspecified) detection limit, it appears that we must rely prime rily on removing graphite samples from the reactor and measuring concentration using a mass spectrometer.

2. One could, of course, use a mass spectrometer to determine this isotopic ratio. Fo uranium hexafluoride $\left(\mathrm{UF}_{6}\right)$, another possibility is to use the rate of neutron emissior which is very sensitive to the U-234 concentration. Passive neutron counting is fre quently used to determine the enrichment of $U_{6}$, but the difference in the emissio rate between the depleted uranium used in the production of LEU and that used in th. production of HEU is only about three percent. If the depleted UF 6 is stored in larg tanks (US tanks hold about 14 te), a difference this small would be detectable by porta ble instruments with reasonable counting times. See R.H. Augustson and T.D. Reilly "Fundamentals of Passive Nondestructive Assay of Fissionable Material," LA-5651-N (Los Alamos: Los Alamos Scientific Laboratory, 1974) pp. 71-72. Uranium-234 doe not emit gamma rays of sufficient intensity to permit estimates of its concentration.

3. Browne and Firestone give $0.7200 \pm 0.0012$ for the atom fraction of U-235; Cowar and Adler (cited in M. Benedict, T.H. Pigford, and H.W. Levi, Nuclear Chemical Engi neering [New York: McGraw-Hill, 1981] p. 814) give $0.7107 \pm 0.0002$ for the weigh fraction of U-235 in 88 samples of natural uranium.

4. National Council on Radiation Protection and Measurements, Exposure of the Pop. ulations in the United States and Canada from Natural Background Radiation (Bethesda, MD: NCRP Report No. 97, December 1987) p. 49.

5. Cited in NCRP, Natural Background Radiation, p. 49.

6. US enrichment facilities have used irradiated uranium feed, and there is evidence that the Soviet Union has done so as well. See Pacific Northwest Laboratory, PNL4621 Draft, March 1983, pp. 16.4, 17.5; and S. Fetter, T.B. Cochran, L. Grodzins, H.L. Lynch, and M.S. Zucker, "Gamma-Ray Measurements of a Soviet Cruise-Missile Warhead," Science 248 (18 May 1990) p. 829.

7. Techniques based on this idea have been developed to estimate the epithermal-tothermal neutron flux ratio, but these are generally suited to short irradiation times. (See, for example, A. Simonits, F. De Corte, and J. Hoste, "Zirconium as a Multi-isotopic Flux Ratio Monitor and a Single Comparator in Reactor-neutron Activation Analysis," Journal of Radioanalytical Chemistry 31 [1976] pp. 467-486.) All of the target nuclei listed in table A-1 have $I / \sigma_{\text {th }}$ ratios of 0.5 to 3 -differences that are insufficient to permit an accurate estimate of the flux ratio (where $I=$ resonance capture integral, and $\sigma_{\text {th }}=$ cross-section for thermal capture). Target nuclei with $I / \sigma_{\text {th }}>10$ that produce radionuclides with half-lives greater than one year include Cd-108, Cs-133, Gd-152, Ho-165, and Pt-192.

8. A. de la Garza, G.A. Garrett, and J.E. Murphy, "Multicomponent Isotope Separa- 
tion in Cascades," Chemical Engineering Science 15 (3-4) (September 1961) pp. 188209.

9. Six fluorine atoms have a mass of $113.990418 \mathrm{amu}$. Other enrichment technologies (e.g., gas centrifuge or aerodynamic separation) will have different, and perhaps much larger, separation factors, but since the separation gains are approximately proportional to the differences in mass, each would have about the same value of $k$.

10. A. de la Garza, G.A. Garrett, and J.E. Murphy, "Multicomponent Isotope Separation in Cascades," Chemical Engineering Science 15 (3-4) (September 1961) p. 198.

11. A. de la Garza, "A Generalization of the Matched Abundance-Ratio Cascade for Multicomponent Isotope Separation," Chemical Engineering Science 18 (1963) pp. 7382. 